\title{
ABCA1 haplodeficiency affects the brain transcriptome following traumatic brain injury in mice expressing human APOE isoforms
}

Emilie L. Castranio, Cody M. Wolfe, Kyong Nyon Nam, Florent Letronne, Nicholas F. Fitz, lliya Lefterov* and Radosveta Koldamova* (D)

\begin{abstract}
Expression of human Apolipoprotein E (APOE) modulates the inflammatory response in an isoform specific manner, with APOE4 isoform eliciting a stronger pro-inflammatory response, suggesting a possible mechanism for worse outcome following traumatic brain injury (TBI). APOE lipidation and stability is modulated by ATP-binding cassette transporter A1 (ABCA1), a transmembrane protein that transports lipids and cholesterol onto APOE. We examined the impact of Abcal deficiency and APOE isoform expression on the response to TBI using 3-months-old, human $A P O E 3^{+/+}\left(E_{3} / \mathrm{AbCa}^{+/+}\right)$and $A P O E 4^{+/+}\left(E 4 / \mathrm{AbCal}^{+/+}\right)$targeted replacement mice, and $\mathrm{APOE3^{+/+ }}$ and $\mathrm{APOE4^{+/+ }}$ mice with only one functional copy of the Abcal gene $\left(E 3 / \mathrm{Abcal}^{+/-} ; \mathrm{E} 4 / \mathrm{AbCa}^{1^{+-}}\right)$. TBl-treated mice received a craniotomy followed by a controlled cortical impact (CCI) brain injury in the left hemisphere; sham-treated mice received the same surgical procedure without the impact. We performed RNA-seq using samples from cortices and hippocampi followed by genome-wide differential gene expression analysis. We found that TBI significantly impacted unique transcripts

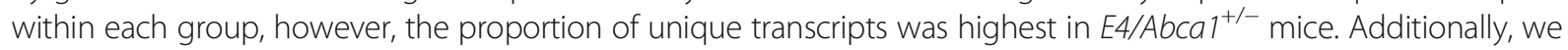
found that Abcal haplodeficiency increased the expression of microglia sensome genes among only APOE4 injured mice, a response not seen in injured APOE3 mice, nor in either group of sham-treated mice. To identify gene networks, or modules, correlated to TBI, APOE isoform and Abcal haplodeficiency, we used weighted gene co-expression network analysis (WGCNA). The module that positively correlated to TBI groups was associated with immune response and featured hub genes that were microglia-specific, including Trem2, Tyrobp, Cd68 and Hexb. The modules positively correlated with APOE4 isoform and negatively to Abca1 haplodeficient mice represented "protein translation" and

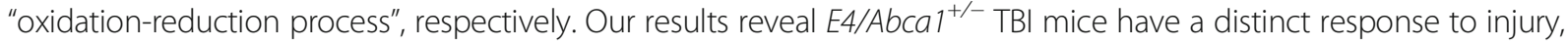
and unique gene networks are associated with $A P O E$ isoform, Abca1 insufficiency and injury.
\end{abstract}

Keywords: Traumatic brain injury, Apolipoprotein E, ABCA1, Transcriptome, WGCNA, Microglia sensome

\section{Introduction}

Traumatic brain injury (TBI) is a significant public health concern; it is a major cause of death and disability in the United States, and its occurrence is highest among multiple vulnerable populations, including the elderly, young adults, and military personnel [16]. No treatment currently exists for the approximately 2 million cases of TBI sustained each year in the United States, and the costs of

\footnotetext{
* Correspondence: iliya@pitt.edu; radak@pitt.edu

Department of Environmental and Occupational Health, University of Pittsburgh, Pittsburgh, PA 15261, USA
}

medical care for 2010 were estimated at $\$ 76.5$ billion annually [10].

TBI is caused by an initial external force, whether a physical object or inertia, contacting the head [27]. The impact and initial mechanical stress placed on the cells constitute the primary injury, whereas the secondary injury occurs after the inciting traumatic event, and involves multiple pathways and signaling cascades that can cause further damage $[3,13,39]$. Inflammation is a major component of the secondary injury. Inflammation is present in every case of TBI and may be a driving

(c) The Author(s). 2018 Open Access This article is distributed under the terms of the Creative Commons Attribution 4.0 International License (http://creativecommons.org/licenses/by/4.0/), which permits unrestricted use, distribution, and 
force for secondary pathology [13]. Chronic neuroinflammation following TBI was closely associated with neuronal death and impaired cell proliferation in locations both immediately adjacent to, and more distant from, the site of injury [1]. Many studies have shown that levels of inflammation and inflammatory molecules are strongly correlated with multiple measures of outcome in patients, including neurobehavioral impairments and survival rates $[29,45]$. Microglia are the brain's main form of immune response to infection, disease, and injury, as well as the source of inflammation. As such, inflammation and microglia have been recent concentrations of research as a means of developing therapies and improving outcomes of TBI.

Outcomes of TBI include possible changes in cognition, behavior, emotion, and sensory processing, all of which are influenced by injury severity and location [5, 18, 35]. Additionally, research has linked TBI to the risk of developing neurodegenerative diseases, including chronic traumatic encephalopathy and Alzheimer's disease (AD) [36]. The high level of heterogeneity in outcomes suggests a significant role for genetic influence on brain susceptibility and recovery $[15,44]$. The apolipoprotein $\mathrm{E}(A P O E)$ gene has been frequently studied to determine its role in TBI and its isoform-dependent impact on outcome. The $A P O E \& 4$ allele is the strongest genetic risk factor for late onset $\mathrm{AD}$, and is thought to confer worse outcome after TBI [2]. APOE\&4 carriers have been found to have slower recovery, increased risk of posttraumatic seizures, and worse memory performance after TBI $[2,12,14]$. However, multiple studies also show that $A P O E \varepsilon 4$ carriers did not differ from non-carriers in cognitive performance, functional outcomes or recovery after TBI $[11,37]$. The contradictory results so far emphasize the need for more research on APOE and TBI.

APOE is involved in several pathways after a TBI occurs, including inflammation [20]. Inheritance of the $A P O E \varepsilon 4$ allele is associated with increased inflammatory responses, including after TBI [28]. APOE4 may induce a more robust pro-inflammatory reaction from microglia and may suppress anti-inflammatory signaling $[4,23,24,26]$. This may be a result of decreased stability and faster catabolic degradation of APOE4, compared to the other isoforms, which is possibly due to its lower lipidation levels [20]. APOE is secreted as nonlipidated apolipoprotein, cholesterol and phospholipid efflux to lipid-poor APOE is mediated by ATP Binding Cassette Transporter A1 (ABCA1) [7]. Abca1 deficiency results in decreased APOE lipidation and APOE levels [17, 21]. ABCA1 may also play a role in modulating the inflammatory response in the brain. Mice lacking brain ABCA1 saw increased inflammatory gene expression, and the microglia cultured from these mice exhibited an increased pro-inflammatory response, as seen by higher levels of TNF $\alpha$ secretion and lower phagocytic activity, in response to lipopolysaccharide administration [19]. It is not known how Abca1 haploinsufficiency may influence TBI.

We recently performed transcriptional profiling of APOE expressing mice after TBI using Next Generation Sequencing [9]. Using a network-based approach, we were able to identify distinct modules correlated to injury and APOE isoform, as well as a module driven by APOE isoform across TBI groups. The aim of this study was to examine the effect of $A b c a 1$ haploinsufficiency on gene expression induced by TBI in APOE targeted replacement mice using transcriptional profiling and a network-based approach. We used 3-month-old mice expressing human $A P O E 3^{+/+}$and $A P O E 4^{+/+}$isoforms $\left(E 3 / \mathrm{Abcal}^{+/+}\right.$and $E 4 / A b c a 1^{+/+}$, respectively), and compared them to their $A b c a 1$ haploinsufficient counterparts $\left(E 3 / A b c a 1^{+/-}\right.$and $E 4 / A b c a 1^{+/-}$, respectively), after performing a controlled cortical impact. Transcriptional profiling of hippocampal and cortical tissue from the injury site was performed using RNA-sequencing (RNA-seq). $E 4 / \mathrm{Abcal}^{+/-}$mice had higher expression levels of the common up-regulated transcripts after TBI, which included genes related to the immune response and inflammatory response. We then examined how ABCA1 insufficiency impacted expression of the microglia sensome genes, and found that $E 4 / \mathrm{Abcal}^{+/-}$TBI mice expressed these genes higher than $E 4 / A b c a 1^{+/+}$TBI mice, whereas no difference was found when comparing sham $\mathrm{Abcal}^{+/-}$to $\mathrm{Abcal}^{+/+}$ mice of either isoform. There was no effect of $A b c a 1$ haploinsufficiency on the expression of microglia genes in $A P O E 3$ TBI mice. We were able to correlate the transcriptome to each phenotype using a network-based approach, Weighted Gene Co-expression Network Analysis (WGCNA). We found that the immune response module, although correlated positively to all TBI groups regardless of APOE isoform or Abcal copy number, consisted of genes expressed at higher levels in E4/ $A b c a 1^{+/-}$TBI mice, and featured microglia-specific hub genes, including Trem2, Tyrobp, Hexb, and Cd68. Our results demonstrate an effect of ABCA1 deficiency on microglia gene expression after TBI in APOE4 mice.

\section{Materials and methods \\ Animals}

All animal experiments were approved through the University of Pittsburgh Institutional Animal Care and Use Committee and carried out in accordance with PHS policies on the use of animals in research. Human $\mathrm{APOE3}^{+/+}$and $\mathrm{APOE4^{+/+ }}$ targeted replacement mice (referred to as $E 3 / A b c a 1^{+/+}$and $E 4 / A b c a I^{+/+}$) were bred to $A b c a 1^{+/-}$mice to generate $A P O E 3^{+/+} / A b c a 1^{+/-}$and $A P O E 4^{+/+} / \mathrm{Abcal}^{+/-}$(referred to E3/Abca1 ${ }^{+/-}$and E4/ $A b \mathrm{CaI}^{+/-}$, respectively) [8, 17]. All mice were on the C57BL/6 genetic background and experimental groups 
consisted of both genders. Experimental mice were kept on a $12 \mathrm{~h}$ light-dark cycle with ad libitum access to food and water. At 3 months of age, these mice were randomly assigned to either sham or controlled cortical impact (CCI) experimental group. Mice were handled for 2 days ( 5 min per day) prior to surgical procedures. All materials were purchased through ThermoFisher Scientific, unless otherwise noted.

\section{Traumatic brain injury}

CCI model of brain injury was performed as previously described [9]. Anesthesia was induced using 5\% isoflurane, after which it was maintained at $1.5 \%$ isoflurane. The head was secured using a stereotaxic frame, and core body temperature was held at $37^{\circ} \mathrm{C}$ using a heating pad. After shaving the heads, two separate iodine - alcohol washes were performed to sterilize the surgical site. A 50\% mixture of bupivacaine and lidocaine was applied to the area and ophthalmic ointment was applied to the eyes. The scalp was opened with a midline incision exposing the dorsal aspect of the skull and the skull leveled. A $4.5 \mathrm{~mm}$ diameter craniotomy was performed over the left parietal cortex using a dental drill. Once the bone flap was removed, mice in the $\mathrm{CCI}$ group received a single impact at $1.0 \mathrm{~mm}$ depth with a $3.0 \mathrm{~mm}$ diameter metal tip onto the cortex (3 m/s, $100 \mathrm{~ms}$ dwell time; Impact One, Leica). Sham mice received identical anesthesia and craniotomy, but did not receive impact and are considered negative controls. Following the impact, the surgical site was sutured, triple antibiotic cream applied, Buprenex $(0.1 \mathrm{mg} / \mathrm{kg}$, IP) provided for analgesia, and sterile saline administered for rehydration. Mice were allowed to recover on heating pad, until freely mobile, before returning to their home cage.

\section{Tissue processing}

Fourteen days post-injury, mice were anesthetized using Avertin $(250 \mathrm{mg} / \mathrm{kg}$ of body weight, i.p.) and perfused transcardially with $20 \mathrm{~mL}$ of cold $0.1 \mathrm{M} \mathrm{PBS} \mathrm{pH} \mathrm{7.4}$ $[9,31]$. Brains were rapidly removed and a $1.5 \mathrm{~mm}$ coronal section of the brain, including the injury site, taken by slicing the brain at $-2.5 \mathrm{~mm}$ and $-4.0 \mathrm{~mm}$ from bregma. Within the coronal slice, the hemispheres were separated, and the subcortical tissue was dissected out; hippocampal and cortical tissue were snap-frozen together for RNA isolation and RNA-seq.

\section{RNA isolation and RNA sequencing}

All procedures were performed as before [9, 32]. CCI and sham mice consisting of both genders for each genotype were used for RNA-seq. RNA was isolated from frozen cortices and hippocampi at the injury site and purified using RNeasy kit (Qiagen) according to the manufacturer recommendations. Quality control of all
RNA samples was performed on a 2100 Bioanalyzer instrument (Agilent Technologies) and samples with RIN $>8$ were further used for library construction using RNA Library Prep Reagent Set (Illumina). Libraries for Next Generation Sequencing were generated by PCR enrichment including incorporation of barcodes to enable multiplexing. Sequencing was performed by the Next Generation Sequencing Center (University of Pennsylvania, https://ngsc.med.upenn.edu/) on HiSeq 2500 machine. Following initial processing and quality control, the sequencing datasets were further analyzed for differential gene expression, which in all cases was calculated using Subread/featureCounts (v1.5.0; https:// sourceforge.net/projects/subread/files/subread-1.5.0/) for read alignment and summarization and statistical package edgeR (v3.14.0; https://bioconductor.org/packages/ release/bioc/html/edgeR.html). Lists of differentially expressed genes are further analyzed as described in the following section.

\section{Weighted gene co-expression network analysis (WGCNA)} Network analysis was performed using WGCNA (v.1.51; https://cran.r-project.org/web/packages/WGCNA/index. html) $[33,48]$. Libraries are clustered by gene expression enabling the detection of outliers and the power is determined by scale free topology model. Modules were generated automatically using a soft thresholding power, $\beta=10$, a minimum module size of 18 genes and a minimum module merge cut height of 0.25. Modules were named by conventional color scheme and then correlated with trait data using Pearson's correlation (APOE isoform, Injury, Abca1 copy number). Statistical significance was determined by student's $t$-test, $p<0.05$. All modules were summarized by module eigengenes (ME), the first principle component of each module that was calculated as a synthetic gene representing the expression profile of all genes within a given module.

Representative networks were built using hub genes and the transcripts connected to them. Hub genes were identified using cutoffs of their interconnectivity within the module (module membership, $>0.8$ ), and the correlation between expression level and trait (gene significance, $>0.2$ ). Once the hub genes are selected, the connections to other transcripts are sorted by weight, with the first 150 connections used for visualization. Gene-association networks of interest were visualized using Cytoscape (v3.3.0). Unsupervised hierarchical clustering was performed on ME turquoise using pheatmap (v1.0.10; https:// cran.r-project.org/web/packages/pheatmap/index.html) to identify 2 sub-modules. A representative network was built for each sub-module consisting of only genes within the sub-module. 


\section{Functional pathway analysis}

Functional annotation clustering was performed using the Database for Annotation, Visualization and Integrated Discovery (DAVID v6.8, https://david.ncifcrf.gov) [25].

\section{Results}

TBI induces changes to the transcriptome that are common among both $\mathrm{AbCa}^{+/+}$and $\mathrm{AbCa1}^{+/-}$mice expressing human APOE isoforms

To examine the effect of TBI on gene expression in the brains of $A b c a 1^{+/-}$and $A b c a 1^{+/+}$mice expressing human APOE isoforms $\left(E 3 / A b c a 1^{+/-}, E 4 / A b c a 1^{+/-}, \mathrm{E} / \mathrm{Abcal}^{+/-}\right.$, $\left.E 4 / \mathrm{Abcal}^{+/+}\right)$, we collected hippocampal and cortical tissue from the injury site at 14 days post-injury. Total RNA was isolated from these tissues and used for RNA-sequencing. As shown in Fig. 1a-d, TBI significantly affected the transcriptome within each genotype. We highlighted several genes of interest on the scatterplots increased by TBI, and while they were differentially expressed within all the groups, the group with the highest CPM values was the E4/Abca1 ${ }^{+/-}$TBI mice. To determine what similarities existed among the affected biological processes, we examined the differentially expressed genes that were significant and common among the 4 genotypes. The expression levels of the top 100 upand down-regulated genes are shown in the heatmap (Fig. 1e). Although the genes are common, the E4/ $A b c a 1^{+/-}$mice show the highest expression levels of the upregulated genes. Figure if shows the biological processes associated with the common, upregulated genes. There were 1196 up-regulated genes common to all the groups and the top Gene Ontology (GO) terms derived from these genes were "immune system process", "innate immune response", and "inflammatory response". In comparison, Fig. $1 \mathrm{~g}$ shows the biological processes associated with the common, downregulated genes. There were 579 downregulated genes common to the groups, and these genes were functionally associated with "regulation of ion transmembrane transport", and "potassium ion transport".

TBI significantly alters the expression of transcripts unique to each genotype, with the highest proportion of unique transcripts among $\mathrm{E} 4 / \mathrm{AbCa1^{+/- }}$ mice

We were interested in whether the response to TBI was specifically influenced by each genotype, particularly by Abcal haplodeficiency in conjunction with APOE4 isoform. To do this, we determined the proportion of genes that were differentially expressed, either in common among several of the groups or were uniquely expressed in only one group. These proportions are shown for each genotype in the donut plots in Fig. 2. As seen in Fig. 2a, $E 4 / \mathrm{Abca1}^{+/-}$mice have a higher proportion of unique transcripts $(26 \%)$ that are up-regulated by TBI than the other genotypes $\left(E 3 / A b c a 1^{+/-}\right.$: $5.5 \%$; E3/Abca1 $1^{+/+}$: $10 \%$;
E4/Abcal $\left.{ }^{+/+}: 5.0 \%\right)$. The biological processes derived from the unique genes of the $E 4 / A b c a 1^{+/-}$mice, include "positive regulation of neuroblast differentiation" and "positive regulation of apoptotic process". The biological functions associated with the unique, upregulated genes within each group differ greatly; the other top terms include "determination of left/right symmetry", "negative regulation of cell proliferation", and "inner dynein arm assembly" for $\mathrm{E} 3 / \mathrm{Abcal}^{+/-}, \mathrm{E} 3 / \mathrm{Abca1^{+/+ }}, \mathrm{E} 4 / \mathrm{Abca1^{+/+ }}$ respectively. Expression plots show the distinct upregulation in $E 4 / A b c a 1^{+-}$TBI mice of several genes, including Plekho1, which has been shown to promote apoptosis (Fig. 2c) [47].

$E 4 / \mathrm{Abca1}^{+/-}$mice, again, have a higher proportion of unique, significant down-regulated transcripts (30\%) than the other genotypes $\left(\mathrm{E}_{3} / \mathrm{Abcal}^{+/-}: 10 \% ; \mathrm{E} / \mathrm{Abcal}^{+/+}: 13 \%\right.$; $E 4 / A b c a 1^{+/+}: 9.4 \%$ ) (Fig. 2b). The top GO terms derived from the unique down-regulated genes for each group are "transcription, DNA-templated", "covalent chromatin modification", "GPI anchor biosynthetic process", and "Protein K63-linked ubiquitination" for E3/Abca1 ${ }^{+/-}$, $E 4 / A b c a 1^{+/-}, E 3 / A b c a 1^{+/+}, E 4 / A b c a 1^{+/+}$, respectively.

\section{Abca1 haploinsufficiency upregulates microglia sensome genes in injured APOE4 mice}

To determine if there was any effect of Abca1 haploinsufficiency on gene expression changes induced by injury, we examined the expression of microglial sensome genes. Although a clear effect of TBI is present in the differential expression of the microglia sensome by $A b c a 1$ genotype, the heatmap also shows that $E 4 / A b c a 1^{+/-}$TBI mice have higher expression levels of microglial sensome genes than the other groups (Fig. 3a). In contrast, there is no effect of $A b c a 1$ copy number on synaptic transmission genes (Fig. 3b), although an injury effect on expression is still visible. We examined the expression levels of the microglia sensome genes within each $A P O E$ isoform, separated by injury status, for the effect of Abcal genotype. Sham mice in both $A P O E$ isoforms (Fig. 3c-d) and injured APOE3 mice (Fig. 3e) have no significant changes in microglia sensome gene expression due to Abca1 haploinsufficiency. In comparison, the injured $\mathrm{E} 4 / \mathrm{Abcal}^{+/-}$mice demonstrate significant expression of the microglia sensome compared to E4/Abca $1^{+/+}$ TBI mice (Fig. 3f). In conclusion, these results demonstrate an effect of $A b c a 1$ haploinsufficiency on the microglia sensome in APOE4 mice after TBI.

\section{WGCNA reveals interconnected gene clusters associated} with each trait of interest- $A P O E$ isoform, Abca1 copy number, and injury status

To identify interconnected gene clusters, or modules, associated within each trait of interest, we employed WGCNA. We were interested in the modules that were 


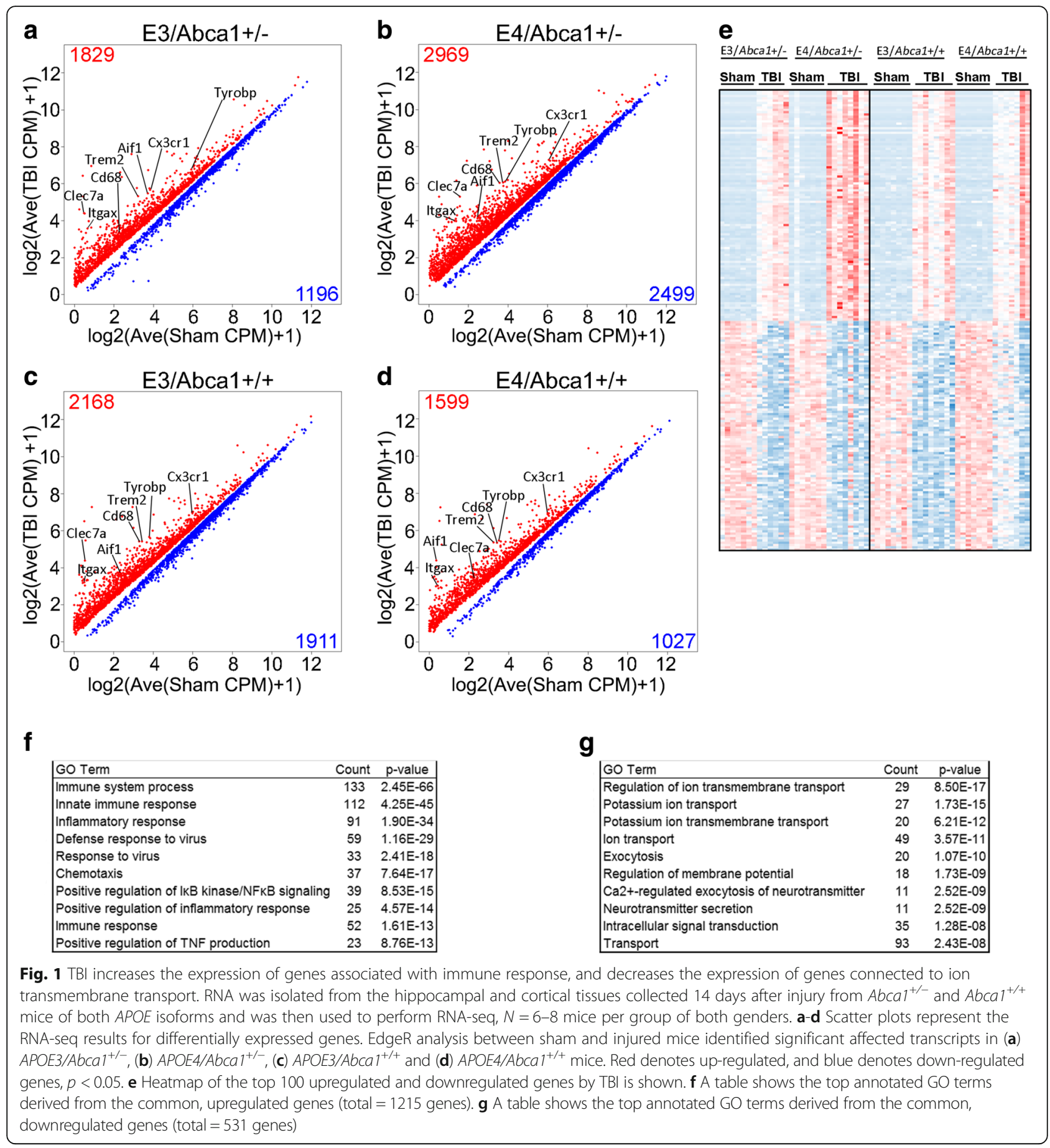

differentially expressed across our traits of interest - injury status, $A P O E$ isoform and $A b c a 1$ copy number. The relationship table (Fig. 4a) shows the MEs of interest and the corresponding correlation coefficients per group.

ME $\tan$ (module size $=182$ genes) correlated across the groups depending on $A P O E$ isoform, regardless of either injury status, or $A b c a 1$ copy number. It positively correlated to APOE4 groups and negatively with APOE3 groups. As seen in the module heatmap (Fig. 4a; far right), the gene members are generally increased in APOE4 mice and decreased in APOE3 mice. The GO terms associated with the module genes were "tRNA aminoacylation for translation", "RNA processing", and "translation". We built a representative network using the hub genes associated with "tRNA aminoacylation for translation", such as Yars, Gars, and Nars, which are aminoacyl-tRNA synthetases.

ME pink correlated with injury status, however, it negatively correlated to TBI groups and positively correlated 


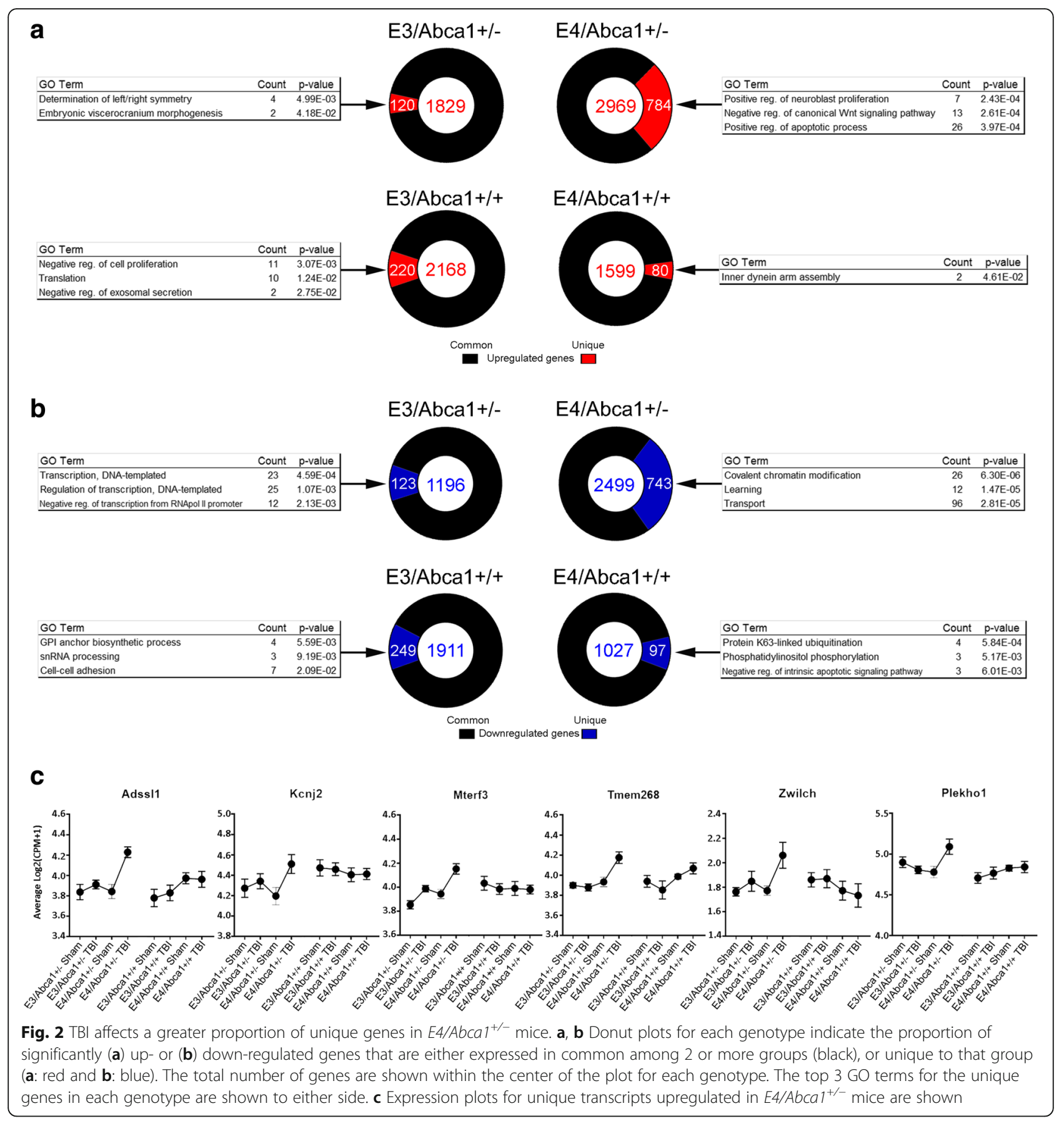

with sham groups. The biological processes associated with ME pink (module size $=518$ genes) included "synaptic vesicle docking", "long-term synaptic potentiation", and "chemical synaptic transmission", which suggests that injury decreases synaptic transmission. The representative network (Fig. 4c) was built around several hub genes related with synaptic transmission, including Stx1a, Snap25, and Lamp5, which are all associated with synaptic vesicle docking and neurotransmitter release. Lamp5, in particular, is associated with GABAergic synaptic transmission and short-term synaptic plasticity [40]. Another hub gene is $P r k c z$, which is necessary for long-term potentiation in hippocampal CA1 pyramidal cells [42].

ME grey60 correlated with $A b c a 1$ copy number, specifically, it negatively correlated with $A b c a 1^{+/-}$mice and positively correlated with $A b c a 1^{+/+}$mice, regardless of injury or $A P O E$ isoform. As seen in Fig. 4d, the network was built around hub genes, which represented GO terms "oxidation-reduction process", "transport", and "aging". These hub genes included a number of the 
a

APOE3 TBI APOE3 Sham APOE4 TBI APOE4 Sham

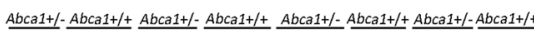

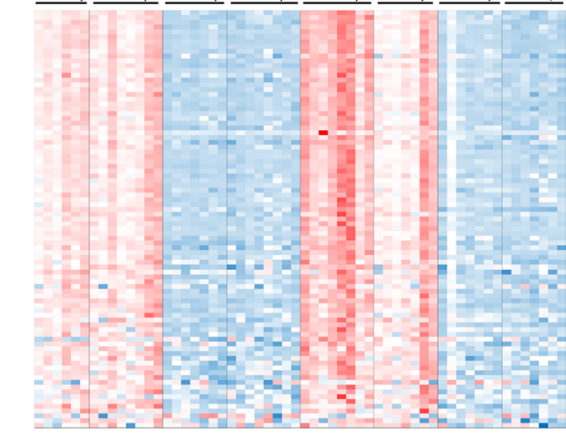

C

APOE3 Sham

Abca1+/-vs Abca1+/+

Log2 Fold Change

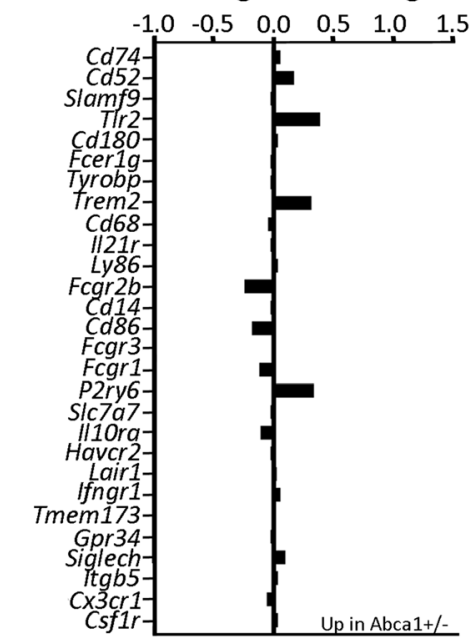

e

APOE3 TBI

Abca1+/+ vs Abca1+/-

Log2 Fold Change

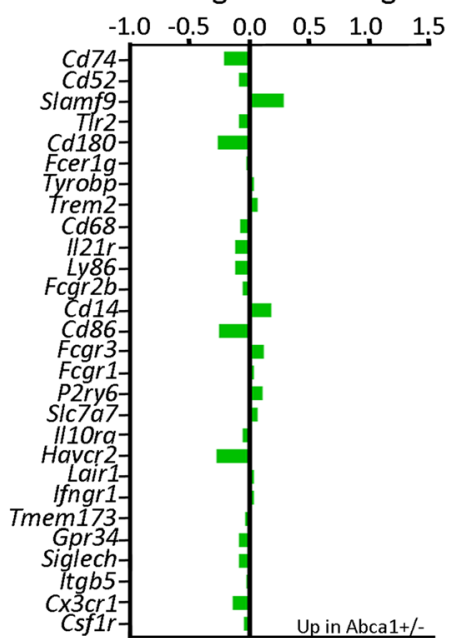

b

APOE3 TBI APOE3 Sham APOE4 TBI APOE4 Sham

Abca1+/- $A b c a 1+/+\underline{A b c a 1+/-}$ Abca1+/+ $A b c a 1+/-\underline{A b c a 1+/+}$ Abca1+/- $A b c a 1+/+$

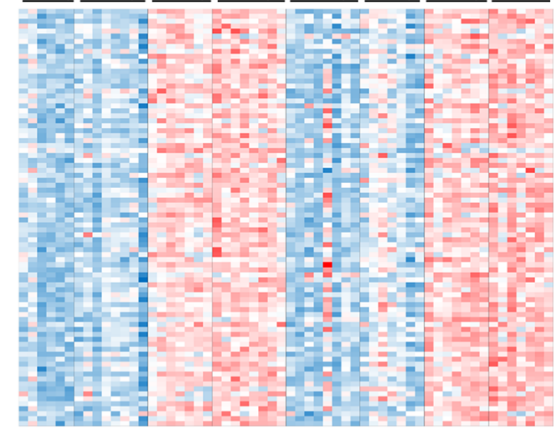

d

APOE4 Sham

Abca1+/- vs Abca1+/+

Log2 Fold Change

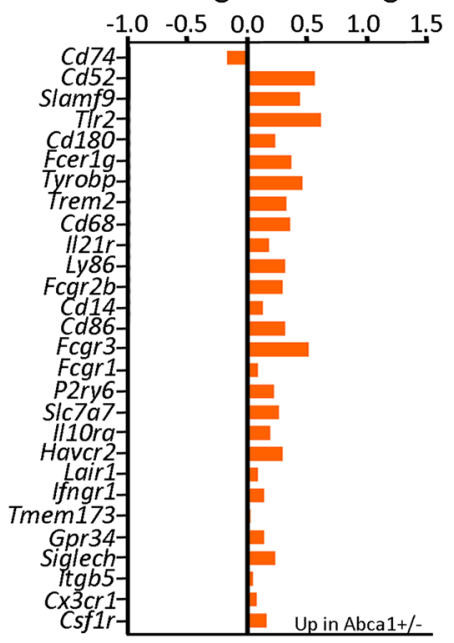

f

APOE4 TBI

Abca1+/+ vs Abca1+/-

Log2 Fold Change

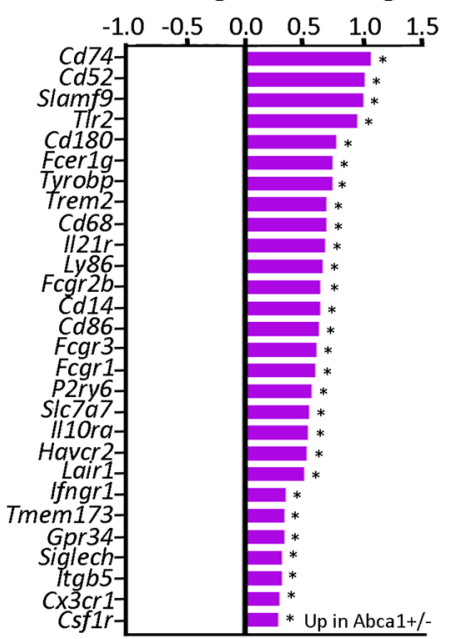

Fig. 3 (See legend on next page.) 
(See figure on previous page.)

Fig. 3 Abcal deficiency affects the microglial response to TBI in an APOE isoform-dependent manner. a-b Heatmaps were generated using normalized $\mathrm{Abcal}^{+/-}$versus $\mathrm{Abcal}{ }^{+/+} \mathrm{CPM}$ values for each group for (a) microglia sensome genes and (b) synaptic transmission genes. Red denotes higher expression values, and blue denotes lower expression values. $n=6-8$ per group, including both males and females. $\mathbf{c}$, e Selected genes from the microglia sensome of $A P O E 3 / A b C a 1^{+/-}$and APOE3/Abcal ${ }^{+/+}$mice are compared separately for (c) sham (black bars) and (e) TBI groups (green bars). Shown are the Log2-fold change values for each gene. $\mathbf{d}$, $\mathbf{f}$ Selected genes from the microglia sensome of APOE $4 / \mathrm{Abcal}^{+/+}$and $A P O E 4 / A b c a 1^{+/-}$mice are compared separately for (d) sham (orange bars) and TBl groups (purple bars). Shown are the Log2-fold change values. *: $p<0.05$

NADH hydrogenase subunits, such as $N D 1, N D 2, N D 4$, ND5 and ND6. Other hub genes were COX1, Atp5j2, and $C Y T B$. All of these hub genes are involved in the mitochondrial respiratory chain [46].

ME turquoise strongly correlated with injury status, however, unlike ME pink, it correlated positively to TBI groups and negatively to sham groups. The genes within ME turquoise are strongly connected and related to the module biological process, as seen in the module membership and gene significance scatterplot (Fig. 5a). Due to the size (module size $=3860$ genes), we were interested in further separating the module. To do this, we ran a pheatmap function on the genes within the module, which aggregates the genes using hierarchical clustering. As shown in Fig. 5b, the pheatmap separated the module into 2 distinct clusters based on injury status and direction of expression. Additionally, the pheatmap shows the expression for all the genes in ME turquoise and the eigengene expression for each sample. The first cluster, Cluster 1 , (size $=2605$ genes) consisted of genes upregulated in TBI groups and downregulated in sham groups. The pheatmap suggests a stronger response of the cluster 1 genes within the $E 4 / A b c a 1^{+/-}$mice, which is consistent with the correlation of ME turquoise to this group in the relationship table. The GO terms derived from Cluster 1 were "immune system response", "innate immune response", and "inflammatory response". Additionally, among the top $10 \mathrm{GO}$ terms was "lipid metabolic process". The representative network (Fig. 5c) was built around hub genes associated with immune response, such as Clec7a, C1qc, and microglia-specific genes, Trem2, Tyrobp, Hexb, and Cd68.

The second cluster, Cluster 2, $($ size $=1111$ genes $)$ featured genes downregulated in the TBI mice, upregulated in the sham mice. Functionally, this cluster is enriched in genes connected to the GO term "transport", other transport-associated terms, such as "vesicle-mediated transport", but also GO terms "sterol biosynthetic process" and "cholesterol biosynthetic process". The network (Fig. 5d) built for Cluster 2 excluded any genes from Cluster 1, and functionally represents transport, however, while hub genes, Gabrb2, Gabrg2, and Atp1b1 all relate directly to the transport of ions across the membrane, through this mechanism, these genes are also strongly associated with synaptic transmission. In conclusion, ME turquoise strongly correlated to injury status, but hierarchical clustering of the genes revealed two distinct clusters associated with the gene expression direction. Cluster 1 was larger and featured genes related to immune response and was more strongly upregulated in $\mathrm{E} 4 / \mathrm{Abca1}^{+/-}$mice, while cluster 2 featured genes downregulated in TBI groups and represented transport, but functionally are also involved in synaptic transmission.

\section{Discussion}

We examined the impact of $A b c a 1$ deficiency and $A P O E$ isoform expression on the response to traumatic brain injury. Our goal was to identify differences in the transcriptional response and trait-associated genome-wide correlated gene networks between $\mathrm{Abcal}^{+/+}$and $\mathrm{Abcal}^{+/-}$mice following a controlled cortical impact in human $A P O E 3^{+/}$ ${ }^{+}$and $A P O E 4^{+/+}$targeted replacement mice. We found that the four groups within our study $-E 3 / A b c a 1^{+/}$, $E 4 / \mathrm{Abcal}^{+/-}, E 3 / A b c a 1^{+/+}$, and E4/Abca1 $1^{+/+}$- had common and distinct responses to TBI. E4/Abca1 ${ }^{+/-}$mice had the highest proportion of unique transcripts affected by TBI, suggesting that $E 4 / A b c a 1^{+/-}$mice are more disposed to changes in gene expression by TBI than the other groups, and demonstrate possible pathways that could be associated with worsened outcome, such as downregulated genes associated with learning. The common, up-regulated genes were associated with biological processes related to immune response, innate immune response and inflammatory response. While, these genes were common among the four groups, the E4/Abcal ${ }^{+/-}$ mice had higher expression levels of the genes upregulated by TBI compared to the other groups, suggesting a role for $A P O E$ isoform and ABCA1 in the expression of inflammatory genes after TBI. Consequently, we examined the effect of $A b c a 1$ insufficiency on microglia sensome genes by injury status and APOE isoform. When comparing injured $A b c a 1^{+/-}$to $A b c a 1^{+/+}$mice, we found $E 4 / \mathrm{Abcal}^{+/-}$TBI mice had increased expression of the microglia sensome genes. In contrast, there was no effect of Abca1 copy number in $A P O E 3$ mice, sham or TBI. These results suggest that $A b c a 1$ haploinsufficiency may influence the inflammatory response following TBI, particularly through an impact on microglia and their gene expression. This effect is seen only among APOE4 mice, not $A P O E 3$ mice; this response may be related to the isoform-specific effect on inflammation. Additionally, the APOE4 isoform may be more vulnerable to the 


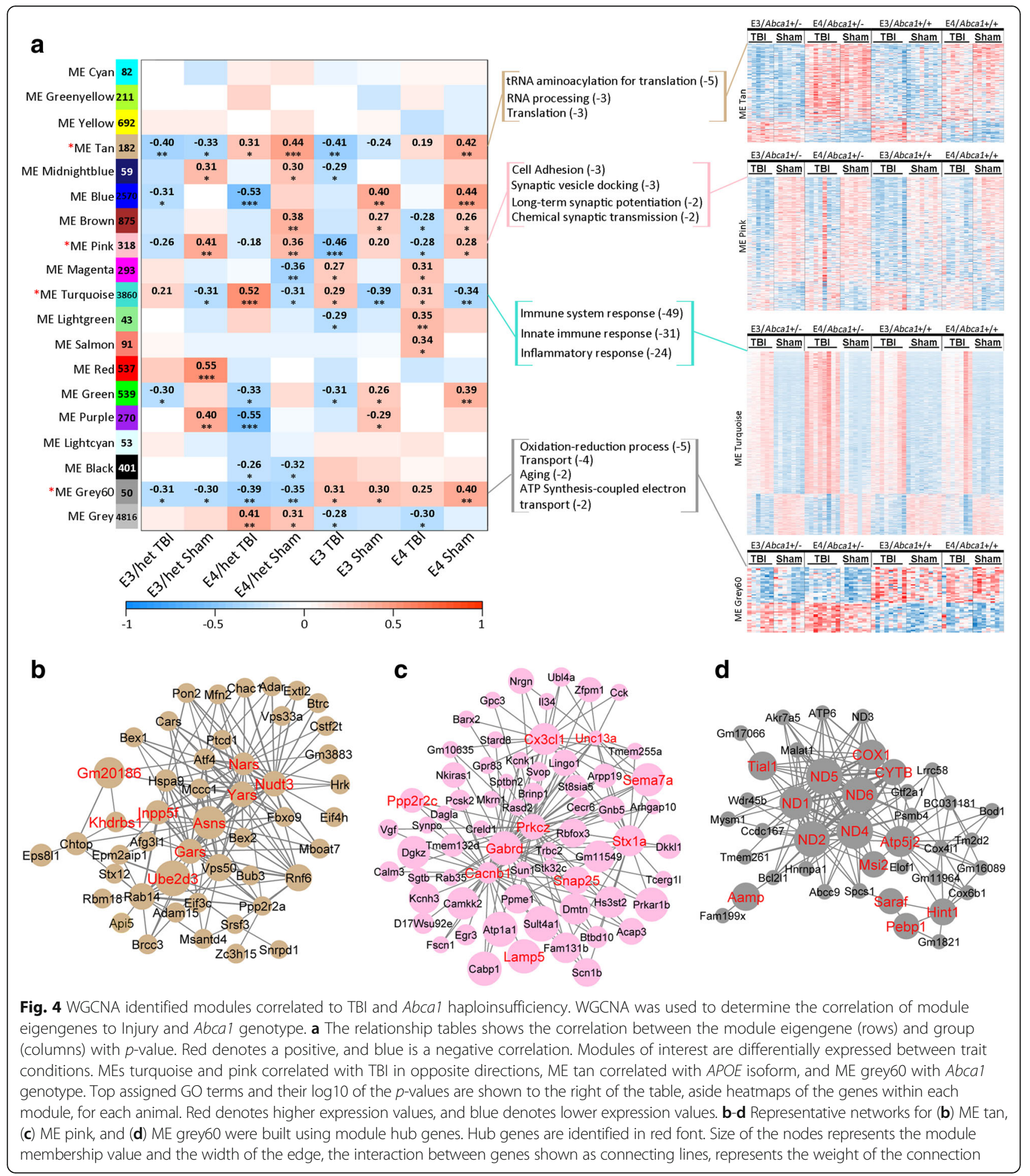

consequences of Abca1 haploinsufficiency due to a gene-gene interaction, a result also demonstrated by data from AD-model mice [17]. These results suggest a possible mechanism for worse outcome after TBI associated with $A P O E 4$ isoform.
Using WGCNA, we identified modules associated with each trait - injury, $A P O E$ isoform and $A b c a 1$ copy number. ME tan was associated with $A P O E$ isoform; the module positively correlated with $A P O E 4$ mice and negatively correlated with $A P O E 3$ mice, regardless of Abca1 copy 

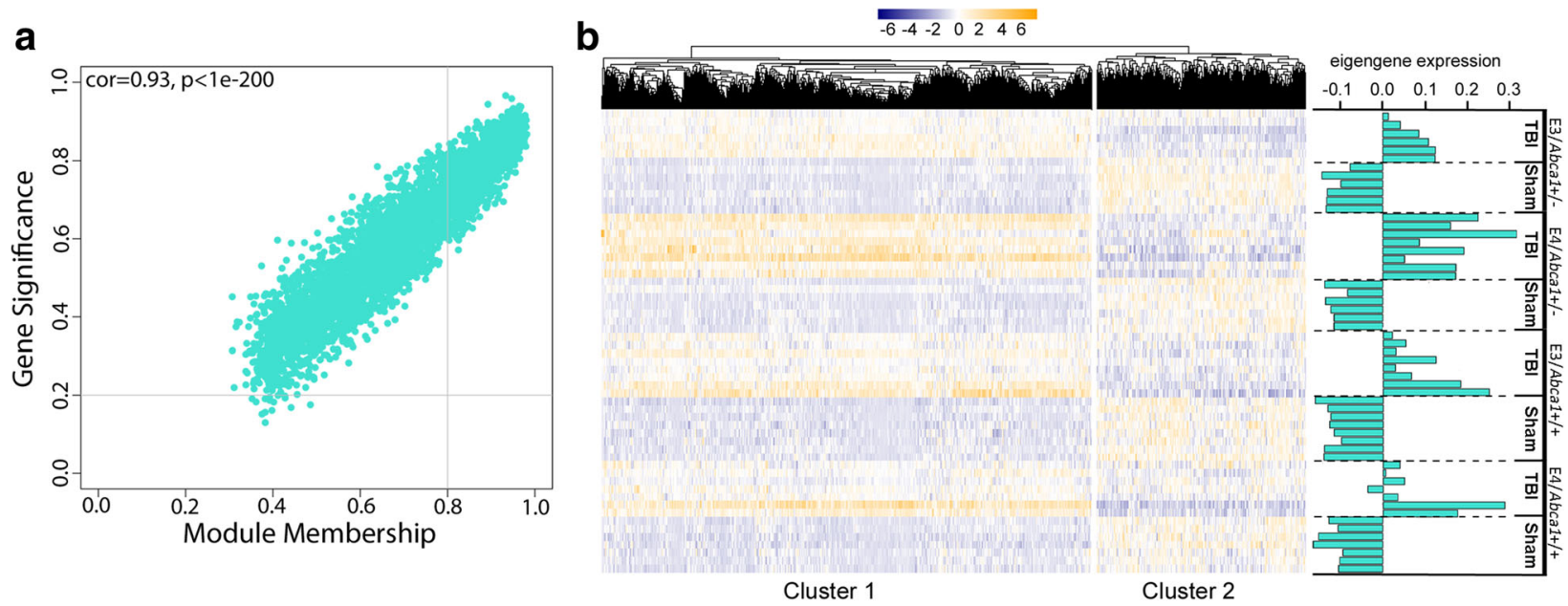

C

\begin{tabular}{lcc|} 
GO Term & Count & p-value \\
\hline Immune system process & 177 & $9.01 E-61$ \\
Innate immune response & 152 & $2.16 E-39$ \\
Inflammatory response & 126 & $6.91 E-31$ \\
Defense response to virus & 71 & $6.13 E-22$ \\
Positive regulation of IKB kinase/NFKB signaling & 60 & $2.54 E-17$ \\
Response to virus & 40 & $1.45 E-14$ \\
Regulation of cell proliferation & 72 & $4.00 E-14$ \\
Positive regulation of NFKB transcription factor activity & 44 & $7.21 E-13$ \\
Chemotaxis & 46 & $8.76 E-13$ \\
Lipid metabolic process & 112 & $1.44 E-12$ \\
\hline
\end{tabular}

d

\begin{tabular}{lcc|} 
GO Term & Count & $p$-value \\
\hline Transport & 157 & $1.02 \mathrm{E}-11$ \\
Intracellular protein transport & 36 & $5.44 \mathrm{E}-09$ \\
Vesicle-mediated transport & 29 & $3.08 \mathrm{E}-06$ \\
Sterol biosynthetic process & 10 & $4.03 \mathrm{E}-06$ \\
Positive regulation of protein targeting & 16 & $9.00 \mathrm{E}-06$ \\
Protein transport & 55 & $1.66 \mathrm{E}-05$ \\
Potassium ion transport & 20 & $1.88 \mathrm{E}-05$ \\
Cholesterol biosynthetic process & 10 & $1.92 \mathrm{E}-05$ \\
lon transport & 54 & $2.30 \mathrm{E}-05$ \\
Membrane fusion & 10 & $4.22 \mathrm{E}-05$ \\
\hline
\end{tabular}
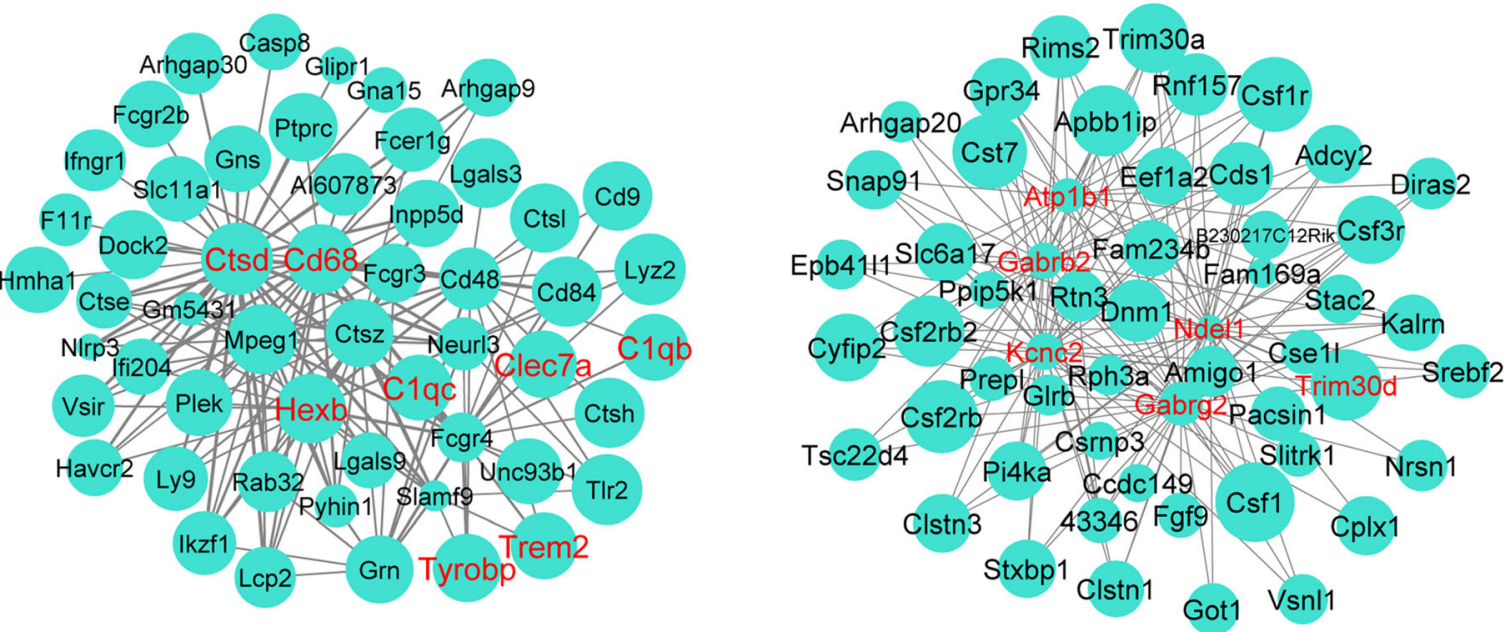

Fig. 5 ME turquoise consists of two sub-modules correlated to injury status. a Scatter plot for ME turquoise module showing the correlation between module membership and gene significance. The degree of association between MM and GS was evaluated by Pearson correlation. Correlation value and $p$ value are indicated in the plot. $\mathbf{b}$ The pheatmap shows normalized gene expression values beside module eigengene expression values for each sample for ME turquoise. The pheatmap function aggregated the module into 2 sub-modules (Cluster 1 and Cluster 2) by hierarchical clustering. c-d Tables of top assigned GO terms are shown above representative networks for (c) cluster 1 and (d) cluster 2 . Hub genes are identified in red font. Size of the nodes represents the module membership value and width of the edge represents the weight of the connection

number or injury status. The representative network was associated with the GO term "tRNA aminoacylation for translation", and included hub genes Yars, Gars, and Nars, which are aminoacyl-tRNA synthetases. Mutations in these genes are associated with Charcot-Marie-Tooth disease, one of the most commonly inherited neurological disorders [6]. Additionally, a metabolomics study on AD patient CSF and plasma found that a pathway significantly affected in plasma by AD severity was aminoacyl-tRNA biosynthesis, however, the mechanisms associated with altered aminoacyl-tRNA synthetases and AD remain unknown [41]. 
The "synaptic transmission" module, ME pink was significantly correlated and down-regulated by injury across the groups. The network represented GO terms "synaptic vesicle docking", "long-term synaptic potentiation", and "chemical synaptic transmission". The hub genes featured in the representative network, included Stx 1a, Snap25, and Lamp5, which are all associated with synaptic vesicle docking and neurotransmitter release. Lamp5 localizes in the synapse, where it may play a regulatory role in GABAergic synaptic transmission [40]. Another hub gene in this network, Prkcz, has an important role in hippocampal long term potentiation and learning [42]. Its expression mediates the storage of specific forms of long term memory [38]. The negative association between this network and injury is consistent with the impact that TBI is known to have on memory.

The network representing ME grey60 was associated with "oxidation-reduction process" and "aging". This module was differentially expressed dependent on Abca1 copy number; the module was downregulated in $A b c a 1^{+/-}$mice, and upregulated in $A b c a 1^{+/+}$mice. The network was built around hub genes involved in the mitochondrial respiratory chain, including a number of the NADH hydrogenase subunits, such as ND1, ND2, ND4, ND5 and ND6, as well as, COX1, Atp5j2, and CYTB. Mitochondrial dysfunction and dysfunctional energy metabolism are early pathological features of multiple neurological diseases, including Alzheimer's disease, Parkinson's disease and Huntington's disease $[34,46]$. Perturbations in the mitochondrial respiratory chain results in decreased ATP synthesis, the generation of free radicals and oxidative damage resulting in neuronal dysfunction and apoptosis [30]. HDL and HDL-associated lipids play key roles in the regulation and preservation of mitochondrial function [43]. ABCA1 is an essential mediator of HDL formation, which may explain the negative correlation between $\mathrm{Abcal}^{+/-}$mice and this network.

ME turquoise correlated with the groups by injury status, however, the module separated into distinct gene clusters representing unique biological processes. Using the pheatmap function, we were able to separate ME turquoise into 2 sub-modules by hierarchical clustering. The clusters were separated based on injury status and the direction of gene expression. The first cluster was larger and consisted of genes upregulated by injury. This cluster represented the "immune response" and the network was built from several microglia-specific genes including Trem2, Tyrobp, Hexb, and Cd68. Although there was no specific modulatory effect of $A P O E$ isoform or $A b c a 1$ copy number on the module, the expression of the module genes was much higher in $E 4 / A b c a 1^{+/-}$injured mice, which is consistent with our other results.

ABCA1 is a major regulator of cholesterol transport and an essential mediator of high density lipoprotein generation [22]. ABCA1 may have a crucial role in the response to TBI by providing essential cholesterol and phospholipids required for repair. However, ABCA1 may also influence the TBI response through its modulatory effects on the inflammatory response. Mice lacking brain ABCA1 exhibit increased neuroinflammation, and in particular have an increased microglial pro-inflammatory response [19]. The effect of ABCA1 on inflammation could also occur through its functional role in mediating cholesterol efflux onto lipid-poor apolipoprotein, including APOE. It was previously shown that the loss of ABCA1 function results in a reduction of APOE, and data from experimental animals show that $A b c a 1$ deficiency abolishes the lipidation of APOE [21]. The isoform-dependent effect of APOE is possibly driven by lipidation status, which has been shown to affect its stability and degradation rate. Our study shows that ABCA1 haploinsufficiency increased expression of the microglia sensome genes in an APOE isoform dependent manner, which suggests gene-gene interactions as a possible mechanism for worsened outcomes after TBI in APOEE4 carriers.

\section{Conclusions}

Our results suggest a possible role for $A b c a 1$ haplodeficiency on the response to TBI in APOE4 TBI mice at a transcriptional level. When we compared $\mathrm{Abca1}^{+/+}$mice to $A b c a 1^{+/-}$mice by injury status and isoform, we found that the lack of one copy of Abca1 significantly increased the expression of microglia sensome genes only in APOE4 TBI mice. This was consistent with the higher expression of the common, upregulated genes, which were associated with immune response. Furthermore, $E 4 / \mathrm{Abcal}^{+/-}$showed the highest expression of the immune response gene network, which also included microglia-specific hub genes, Trem2, Tyrobp, Hexb, and Cd68. Our results suggest that gene-gene interactions can modify the response of $A P O E 4$ mice to harmful effects.

\section{Abbreviations \\ ABCA1: ATP Binding Cassette Transporter A1; AD: Alzheimer's disease; APOE: Apolipoprotein E; CCl: Controlled Cortical Impact; GO: Gene Ontology; ME: Module Eigengene; RNA-seq: RNA-sequencing; TBI: Traumatic brain injury; WGCNA: Weighted Gene Co-expression Network Analysis}

\section{Acknowledgements \\ The authors would like to acknowledge the excellent assistance of Dr. Anais Mounier for her assistance in library generation. We would also like to acknowledge the assistance of the lab technicians Brittany Playso, and Heather Wells, who were responsible for the management of the animal colony during the course of the project.}

\section{Funding}

This study was supported by the National Institutes of Health (ES024233, AG056371, AG057565 and K01AG044490) and the U.S. Department of Defense (W81XWH-13-1-0384). 


\section{Availability of data and materials}

The RNA-seq data that support the findings of this study are available in the NCBI GEO repository, with accession code GSE117223 (https:/www.ncbi.nlm.nih.gov/geo/query/acc.cgi?acc=GSE1 17223).

\section{Authors' contributions}

ELC was a major contributor to all the experimental work, data analysis, and manuscript writing. FL generated the libraries for RNA-seq. CMW, KNN, NFF, IL and RK were involved in data analysis. NFF, RK and IL were involved in project administration. RK and IL conceptualized the project, acquired funding and assisted with manuscript development. All authors read and approved the final manuscript.

\section{Ethics approval and consent to participate}

All animal experiments were approved through the University of Pittsburgh Institutional Animal Care and Use Committee and carried out in accordance with PHS policies on the use of animals in research.

\section{Consent for publication}

Not applicable.

\section{Competing interests}

The authors declare that they have no competing interests.

\section{Publisher's Note}

Springer Nature remains neutral with regard to jurisdictional claims in published maps and institutional affiliations.

Received: 12 June 2018 Accepted: 12 July 2018

Published online: 26 July 2018

\section{References}

1. Acosta SA, Tajiri N, Shinozuka K, Ishikawa H, Grimmig B, Diamond DM et al (2013) Long-term upregulation of inflammation and suppression of cell proliferation in the brain of adult rats exposed to traumatic brain injury using the controlled cortical impact model. PLoS One 8:e53376. https://doi. org/10.1371/journal.pone.0053376

2. Alexander S, Kerr ME, Kim Y, Kamboh MI (2007) Apolipoprotein E4 allele presence and functional outcome after severe traumatic brain injury. J Neurotrauma 24:790-797

3. Anthonymuthu TS, Kenny EM, Bayir H (2016) Therapies targeting lipid peroxidation in traumatic brain injury. Brain Res 1640:57-76. https://doi.org/ 10.1016/j.brainres.2016.02.006

4. Barger SW, Harmon AD (1997) Microglial activation by Alzheimer amyloid precursor protein and modulation by apolipoprotein E. Nature 388:878-881. https://doi.org/10.1038/42257

5. Bazarian JJ, Cernak I, Noble-Haeusslein L, Potolicchio S, Temkin N (2009) Long-term neurologic outcomes after traumatic brain injury. J Head Trauma Rehabil 24:439-451. https://doi.org/10.1097/HTR.0b013e3181c15600

6. Blocquel D, Li S, Wei N, Daub H, Sajish M, Erfurth ML et al (2017) Alternative stable conformation capable of protein misinteraction links tRNA synthetase to peripheral neuropathy. Nucleic Acids Res 45:8091-8104. https://doi.org/ 10.1093/nar/gkx455

7. Brooks-Wilson A, Marcil M, Clee SM, Zhang LH, Roomp K, van Dam M et al (1999) Mutations in ABC1 in tangier disease and familial high-density lipoprotein deficiency. Nature Genet 22:336-345

8. Carter AY, Letronne F, Fitz NF, Mounier A, Wolfe CM, Nam KN et al (2017) Liver $X$ receptor agonist treatment significantly affects phenotype and transcriptome of APOE3 and APOE4 Abca1 haplo-deficient mice. PLoS One 12:e0172161. https://doi.org/10.1371/journal.pone.0172161

9. Castranio EL, Mounier A, Wolfe CM, Nam KN, Fitz NF, Letronne F et al (2017) Gene co-expression networks identify Trem2 and Tyrobp as major hubs in human APOE expressing mice following traumatic brain injury. Neurobiol Dis 105:1-14. https://doi.org/10.1016/j.nbd.2017.05.006

10. Centers for Disease C, Prevention (2013) CDC grand rounds: reducing severe traumatic brain injury in the United States. MMWR Morb Mortal Wkly Rep 62: 549-552

11. Chamelian L, Reis M, Feinstein A (2004) Six-month recovery from mild to moderate traumatic brain injury: the role of APOE-epsilon4 allele. Brain 127:2621-2628. https://doi.org/10.1093/brain/awh296
12. Crawford FC, Vanderploeg RD, Freeman MJ, Singh S, Waisman M, Michaels L et al (2002) APOE genotype influences acquisition and recall following traumatic brain injury. Neurology 58:1115-1118

13. Das M, Mohapatra S, Mohapatra SS (2012) New perspectives on central and peripheral immune responses to acute traumatic brain injury. J Neuroinflammation 9:236. https:/doi.org/10.1186/1742-2094-9-236

14. Diaz-Arrastia R, Gong Y, Fair S, Scott KD, Garcia MC, Carlile MC et al (2003) Increased risk of late posttraumatic seizures associated with inheritance of APOE epsilon4 allele. Arch Neurol 60:818-822. https://doi.org/10.1001/ archneur.60.6.818

15. Draper K, Ponsford J (2008) Cognitive functioning ten years following traumatic brain injury and rehabilitation. Neuropsychology 22:618-625. https://doi.org/10.1037/0894-4105.22.5.618

16. Faul M, Coronado V (2015) Epidemiology of traumatic brain injury. Handb Clin Neurol 127:3-13. https://doi.org/10.1016/B978-0-444-52892-6.00001-5

17. Fitz NF, Cronican AA, Saleem M, Fauq AH, Chapman R, Lefterov I et al (2012) Abca1 deficiency affects Alzheimer's disease-like phenotype in human ApoE4 but not in ApoE3-targeted replacement mice. J Neurosci 32:13125-13136. https://doi.org/10.1523/JNEUROSCl.1937-12.2012

18. Ghroubi S, Feki I, Chelly H, Elleuch MH (2016) Neuropsychological and behavioral disorders and their correlations with the severity of the traumatic brain injury. Ann Phys Rehabil Med 59S:e134-e135. https://doi.org/10.1016/j. rehab.2016.07.302

19. Karasinska JM, de Haan W, Franciosi S, Ruddle P, Fan J, Kruit JK et al (2013) ABCA1 influences neuroinflammation and neuronal death. Neurobiol Dis 54:445-455. https://doi.org/10.1016/j.nbd.2013.01.018

20. Kim J, Basak JM, Holtzman DM (2009) The role of apolipoprotein E in Alzheimer's disease. Neuron 63:287-303. https://doi.org/10.1016/j.neuron.2009.06.026

21. Koldamova R, Staufenbiel M, Lefterov I (2005) Lack of ABCA1 considerably decreases brain ApoE level and increases amyloid deposition in APP23 mice. J Biol Chem 280:43224-43235. https://doi.org/10.1074/jbc.M504513200

22. Koldamova R, Fitz NF, Lefterov I (2014) ATP-binding cassette transporter A1: from metabolism to neurodegeneration. Neurobiol Dis 72 Pt A:13-21. https://doi.org/10.1016/j.nbd.2014.05.007

23. Laskowitz DT, Fillit H, Yeung N, Toku K, Vitek MP (2006) Apolipoprotein E-derived peptides reduce CNS inflammation: implications for therapy of neurological disease. Acta Neurol Scand (Suppl 185):15-20. https://doi.org/ 10.1111/j.1600-0404.2006.00680.x

24. Li X, Montine KS, Keene CD, Montine TJ (2015) Different mechanisms of apolipoprotein E isoform-dependent modulation of prostaglandin E2 production and triggering receptor expressed on myeloid cells 2 (TREM2) expression after innate immune activation of microglia. FASEB J 29:1754-1762. https://doi.org/10.1096/fj.14-262683

25. Liliang PC, Liang CL, Weng HC, Lu K, Wang KW, Chen HJ et al. (2010) Tau proteins in serum predict outcome after severe traumatic brain injury. J Surg Res 160:302-307. https://doi.org/10.1016/j.jss.2008.12.022

26. Lynch JR, Morgan D, Mance J, Matthew WD, Laskowitz DT (2001) Apolipoprotein E modulates glial activation and the endogenous central nervous system inflammatory response. J Neuroimmunol 114:107-113

27. Maas Al, Stocchetti N, Bullock R (2008) Moderate and severe traumatic brain injury in adults. Lancet Neurol 7:728-741. https://doi.org/10.1016/s14744422(08)70164-9

28. Mannix RC, Zhang J, Park J, Zhang X, Bilal K, Walker K et al (2011) Agedependent effect of apolipoprotein E4 on functional outcome after controlled cortical impact in mice. J Cereb Blood Flow Metab 31:351-361. https://doi.org/10.1038/jcbfm.2010.99

29. Morganti JM, Jopson TD, Liu S, Riparip L-K, Guandique CK, Gupta N et al (2015) CCR2 antagonism alters brain macrophage polarization and ameliorates cognitive dysfunction induced by traumatic brain injury. J Neurosci 35:748-760

30. Muller WE, Eckert A, Kurz C, Eckert GP, Leuner K (2010) Mitochondrial dysfunction: common final pathway in brain aging and Alzheimer's disease--therapeutic aspects. Mol Neurobiol 41:159-171. https://doi.org/10. 1007/s12035-010-8141-5

31. Nam KN, Mounier A, Fitz NF, Wolfe C, Schug J, Lefterov I et al (2016) RXR controlled regulatory networks identified in mouse brain counteract deleterious effects of Abeta oligomers. Sci Rep 6:24048. https:/doi.org/10.1038/srep24048

32. Nam KN, Mounier A, Wolfe CM, Fitz NF, Carter AY, Castranio EL et al (2017) Effect of high fat diet on phenotype, brain transcriptome and lipidome in Alzheimer's model mice. Sci Rep 7:4307. https://doi.org/10.1038/ s41598-017-04412-2 
33. Nam KN, Wolfe CM, Fitz NF, Letronne F, Castranio EL, Mounier A et al (2018) Integrated approach reveals diet, APOE genotype and sex affect immune response in APP mice. Biochim Biophys Acta 1864:152-161. https://doi.org/ 10.1016/j.bbadis.2017.10.018

34. Pathania D, Millard M, Neamati N (2009) Opportunities in discovery and delivery of anticancer drugs targeting mitochondria and cancer cell metabolism. Adv Drug Deliv Rev 61:1250-1275. https://doi.org/10.1016/j. addr.2009.05.010

35. Perez-Garcia G, Gama Sosa MA, De Gasperi R, Lashof-Sullivan M, Maudlin-Jeronimo E, Stone JR et al (2016) Chronic post-traumatic stress disorder-related traits in a rat model of low-level blast exposure. Behav brain res. In: https://doi.org/10.1016/j.bbr.2016.09.061

36. Plassman BL, Grafman J (2015) Traumatic brain injury and late-life dementia. Handb Clin Neurol 128:711-722. https://doi.org/10.1016/B978-0-444-63521-1.00044-3

37. Ponsford J, Rudzki D, Bailey K, Ng KT (2007) Impact of apolipoprotein gene on cognitive impairment and recovery after traumatic brain injury. Neurology 68:619-620. https://doi.org/10.1212/01.wnl.0000254609.04330.9d

38. Shema R, Sacktor TC, Dudai Y (2007) Rapid erasure of long-term memory associations in the cortex by an inhibitor of PKM zeta. Science 317:951-953. https://doi.org/10.1126/science.1144334

39. Simon DW, McGeachy MJ, Bayir H, Clark RS, Loane DJ, Kochanek PM (2017) The far-reaching scope of neuroinflammation after traumatic brain injury. Nat Rev Neurol 13:171-191. https://doi.org/10.1038/nrneurol.2017.13

40. Tiveron MC, Beurrier C, Ceni C, Andriambao N, Combes A, Koehl M et al. (2016) LAMP5 fine-tunes GABAergic synaptic transmission in defined circuits of the mouse brain. PLoS One 11:e0157052. https://doi.org/10.1371/journal. pone. 0157052

41. Trushina E, Dutta T, Persson XM, Mielke MM, Petersen RC (2013) Identification of altered metabolic pathways in plasma and CSF in mild cognitive impairment and Alzheimer's disease using metabolomics. PLoS One 8:e63644. https://doi.org/10.1371/journal.pone.0063644

42. Wang S, Sheng T, Ren S, Tian T, Lu W (2016) Distinct roles of PKCiota/ lambda and PKMzeta in the initiation and maintenance of hippocampal long-term potentiation and memory. Cell Rep 16:1954-1961. https://doi.org/ 10.1016/j.celrep.2016.07.030

43. White CR, Datta G, Giordano S (2017) High-density lipoprotein regulation of mitochondrial function. Adv Exp Med Biol 982:407-429. https://doi.org/10. 1007/978-3-319-55330-6_22

44. Whitnall L, McMillan TM, Murray GD, Teasdale GM (2006) Disability in young people and adults after head injury: 5-7 year follow up of a prospective cohort study. J Neurol Neurosurg Psychiatry 77:640-645. https://doi.org/10. 1136/jnnp.2005.078246

45. Yang SH, Gangidine M, Pritts TA, Goodman MD, Lentsch AB (2013) Interleukin 6 mediates neuroinflammation and motor coordination deficits after mild traumatic brain injury and brief hypoxia in mice. Shock (Augusta, Ga) 40:471-475

46. Yu H, Lin X, Wang D, Zhang Z, Guo Y, Ren X et al (2018) Mitochondrial molecular abnormalities revealed by proteomic analysis of hippocampal organelles of mice triple transgenic for Alzheimer disease. Front Mol Neurosci 11:74. https://doi.org/10.3389/fnmol.2018.00074

47. Zhang L, Xing G, Tie Y, Tang Y, Tian C, Li L et al (2005) Role for the pleckstrin homology domain-containing protein CKIP-1 in AP-1 regulation and apoptosis. EMBO J 24:766-778. https://doi.org/10.1038/sj.emboj.7600532

48. Zhao W, Langfelder P, Fuller T, Dong J, Li A, Hovarth S (2010) Weighted gene coexpression network analysis: state of the art. J Biopharm Stat 20: 281-300. https://doi.org/10.1080/10543400903572753

Ready to submit your research? Choose BMC and benefit from:

- fast, convenient online submission

- thorough peer review by experienced researchers in your field

- rapid publication on acceptance

- support for research data, including large and complex data types

- gold Open Access which fosters wider collaboration and increased citations

- maximum visibility for your research: over $100 \mathrm{M}$ website views per year

At BMC, research is always in progress.

Learn more biomedcentral.com/submissions 